\title{
Thérapie chirurgicale des métastases hépatiques d'un cancer colorectal
}

La résection chirurgicale des métastases hépatiques d'un cancer colorectal, une intervention hautement spécialisée, peut être considérée comme une thérapie appropriée pour prolonger la survie d'un patient et améliorer sa qualité de vie. Telle est la conclusion du conseil d'experts de Swiss Medical Board dans le rapport qui vient d'être approuvé, à condition toutefois qu'un diagnostic soigneux et l'indication d'un tel traitement soient posés par un «Tumor board» interdisciplinaire.

Swiss Medical Board*:

Eva Cignacco ${ }^{a}$,

Peter Jüni ${ }^{b}$, Peter Meier-Abt ${ }^{c}$, Urs Metzger, Nikola BillerAndorno , Stefan Felderf, Brigitte Tagg

a Institut des sciences infirmières, Université de Bâle (Médecine)

b Prof. d'épidémiologie clinique, Université de Berne (Médecine)

c Prof. de pharmacologie clinique, vicerecteur de l'Université de Bâle (Médecine)

d Prof. ém. médecin chef en chirurgie, Zurich (Médecine)

e Prof. d'éthique biomédicale, Université de Zurich (Ethique)

f Prof. d'économie de la santé, Université de Bâle

g Prof. de droit pénal, procé dure pénale et droit de la médecine, Université de Zurich (Droit

* Organisation et composition sous www.medical-board.ch/ index.php?id=818\&L=1

Correspondance:

Susanna Marti Calmell Secrétariat Swiss Medical Board Obstgartenstrasse 21

CH-8090 Zurich

Tél. 0432592479

info[at]medical-board

\section{Résumé du rapport original}

Le terme «cancer (ou carcinome) colorectal (CCR)» englobe les cancers du gros intestin (côlon), du rectum, de l'anus et du canal anal. Chaque année, en Suisse, environ 4000 personnes sont atteintes d'un cancer colorectal. Ce dernier touche surtout les sujets de plus de 70 ans $(56,4 \%$ de cette tranche d'âge) et les personnes âgées de 50 à 69 ans (37,3\% de cette tranche d'âge). Avec quelque 1600 décès par an, le CCR est l'une des principales causes de décès par cancer en Suisse.

Le présent rapport du Swiss Medical Board se penche sur la question de savoir si une résection chirurgicale des métastases hépatiques d'un cancer colorectal offre aux patients concernés un avantage appréciable en termes de survie et de qualité de vie par rapport à d'autres procédés thérapeutiques. Sont également examinés les effets négatifs potentiels des différents procédés thérapeutiques et le rapport coût-efficacité.

Sur la base de la littérature existante, on peut conclure qu'une résection chirurgicale des métastases hépatiques est à conseiller si certains critères sont remplis («métastases résécables d’emblée»). Si les critères de résécabilité ne sont pas remplis, il est encore possible, dans certains cas, de les atteindre secondairement en effectuant au préalable un traitement de réduction tumorale. Le Swiss Medical Board reconnaît cette approche thérapeutique de la «résection secondaire des métastases hépatiques initialement non résécables» comme une méthode prometteuse qu'il convient de poursuivre. Même si les premiers résultats se montrent tout à fait encourageants, il n'est pas encore possible, et serait prématuré, de tirer des conclusions de ces formes de thérapie et des indicateurs prometteurs.

Les connaissances tirées de la présente analyse conduisent aux recommandations suivantes.
- La prise en charge chirurgicale des métastases hépatiques résécables d'emblée dans le respect des critères pertinents de résécabilité est incontestée et doit être réalisée lorsqu'il existe un consentement éclairé du patient.

La prise en charge chirurgicale secondaire des métastases hépatiques résécables après réduction tumorale est une prestation complexe et hautement spécialisée. Ces interventions sont considérées comme des prestations hautement spécialisées par l'Organe de décision de la Convention intercantonale relative à la médicine hautement spécialisée (CIMHS); la désignation définitive des centres compétents n'est pas encore faite. De telles prestations ne doivent être réalisées que dans des centres spécialisés pouvant faire état d'un nombre minimum d'interventions. Dans de tels cas, le chirurgien doit rigoureusement respecter son devoir d'information. On doit viser à un règlement des dépenses uniforme dans toute la Suisse.

Pour chaque patient atteint de métastases hépatiques d'un cancer colorectal, un diagnostic soigneux et une information sur une résécabilité possible doivent être établis. Les patients pour lesquels est posée l'indication d'un tel traitement doivent être évalués par un «tumor board» interdisciplinaire.

- Les patients atteints de métastases hépatiques d'un cancer colorectal qui ne suivent pas un traitement purement palliatif doivent être obligatoirement recensés dans un registre central; ce registre doit être périodiquement évalué et publié pour que l'on puisse identifier le plus rapidement possible les groupes de patients pouvant bénéficier d'une intervention chirurgicale. 